\title{
Acerca da relação entre
}

\section{as Propriedades dos Elementos} e os seus Números Atómicos

\section{- tabelas periódicas e sua evolução}

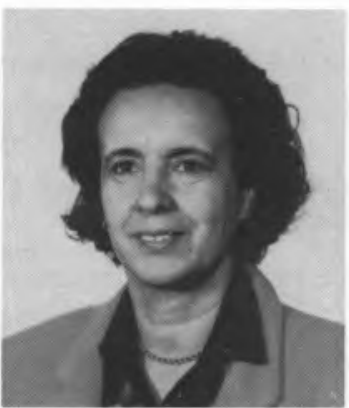

Filomena Camões

Licenciatura em Ciências Físico-Químicas - FCUL (1966).

Doutoramento em Química-Física - Universidade de Newcastle-Upon-Tyne-G.B. (1973).

Professora Associada de Química da Faculdade desde 1979, onde tem regido particularmente cursos de Química Analítica, Electroanalítica e Ambiental.

Responsável pela Linha de Acção «Células Galvânicas» do Centro de Electroquímica e Cinética da Universidade de Lisboa (CECUL) desde a sua fundação em 1975, onde é orientadora e co-orientadora de Doutoramentos e Mestrados. Tem pertencido a diversas Comissôes Directivas do CECUL e foi Secretária do Centro em 1977-78.

Proponente a Coordenadora de Acções de Formação do Fundo Social Europeu para Técnicos Auxiliares de Laboratório.

Responsável por Acções de Formação para Professores do Ensino Secundário sobre Química Analítica e Instrumentação em Química. Membro Associado da Comissão de Química Electroanalítica - V.5 da IUPAC, onde é coordenadora de projectos de investigação científica nesta área. Representante da Sociedade Portuguesa de Química na Working Party of Analytical Chemistry da Federação Europeia das Sociedades de Química.

Delegada em Portugal da EURACHEM.

Membro dos Comités Científicos de diversas conferências científicas internacionais e "Referee» de revistas científicas nacionais e estrangeiras.

Membro das SPQ, SPElectroq., Royal Society of Chemistry $e$ Chemometric Society.

Membro do Conselho Consultivo da Revista de Educação da FCUL.

Membro do Conselho de Gestão do Departamento de Química da FCUL (1976-77).

Membro da Comissão Directiva da FCUL (1977-78).

Presidente da Assembleia de Representantes (1988-89) e da Assembleia Geral de Escola (1988-90) da FCUL.

Autora de mais de 40 trabalhos de índole científica e pedagógica.
Embora possamos encontrar antecedentes de tentativas de agrupar os elementos químicos em função das suas semelhanças e diferenças

Lavoisier - «Tratado Elementar de Química»,

(1789) 23 substâncias elementares

Berzelius - Símbolos químicos; metais e metalóides (1802)

$\begin{array}{ll}\text { Döbereiner } \quad \text { - Três Tríadas: } & \mathrm{Ca}, \mathrm{Ba}, \mathrm{Sr} \\ & \mathrm{Cl} ; \mathrm{Br} ; \mathrm{I} \\ & \mathrm{Li} ; \mathrm{Na} ; \mathrm{K}\end{array}$

Os elementos com comportamento semelhante agrupam-se três a três. Insuficiente para agrupar os 45 elementos já conhecidos à altura.

Dalton - Teoria atómica

(1860) 70 elementos

Charcourtois - Os elementos são ordenados por ordem (1862) e crescente dos pesos atómicos sendo-lhes atriNewlands buído um número a começar em 1.

(1863) São depois divididos sequencialmente por grupos encabeçados pelos sete primeiros elementos então conhecidos; apresenta no entanto incoerências.

$\mathrm{H} \mathrm{Li} \mathrm{Be} \mathrm{B} \mathrm{C} \mathrm{N} \mathrm{O}$

$\mathrm{F} \mathrm{Na} \mathrm{Mg} \mathrm{Al} \mathrm{Si} \mathrm{P} \mathrm{S}$

......

:

Por analogia com as escalas de música, ficou conhecida como a (lei das «oitavas»).

a tabela periódica que usamos hoje em dia resulta do desenvolvimento da classificação elaborada pelo químico russo Dmitri Ivanovich Mendeleev (1834-1907) e pelo físico alemão Lothar Meyer (1830-1895). Embora trabalhando separadamente, desenvolveram sistemas periódicos análogos que publicaram com poucos meses de intervalo: Mendeleev publicou primeiro!

Dmitri Ivanovich Mendeleev nasceu na Sibéria em 1834.

a Centro de Electroquímica e Cinética da Universidade de Lisboa, Dep. de Química, Faculdade de Ciências, Universidade de Lisboa. 
Dada a sua origem viu a sua admissão nas Universidades de Moscovo e de S. Peterburgo interdita. Adquiriu então a qualificação como Professor no Instituto Pedagógico de S. Petersburgo. Colocado a seu pedido em Odessa regressou posteriormente a S. Petersburgo, onde se formou em Química em 1856. Em 1859 o Governo enviou-o para a Universidade de Heidelberg, na Alemanha, onde trabalhou com G. Kirchoff e R. Bunsen.

Começou a estudar os elementos constituintes das substâncias que se nos deparam no dia-a-dia. Descobriu assim propriedades de alguns elementos mais comuns na Natureza, como sejam hidrogénio, carbono, azoto, oxigénio e cloro que lhe serviram de referência no estudo de outros elementos. Em Setembro de 1860 participou no 1. ${ }^{\circ}$ Congresso de Química em Kalsruhe onde estabeleceu importantes contactos com S. Cannizzaro de quem recolheu informações relativamente aos elementos conhecidos, particularmente quanto às diferenças entre valores das massas atómicas e das massas moleculares.

Regressou à Universidade de S. Petersburgo, embora sem cargo fixo, o que veio a alcançar em 1864. Começou por leccionar Química Orgânica, tendo publicado um livro de grande aceitação, sobre o assunto.

Em 1867 foi nomeado Professor de Química Geral tendo sentido a necessidade de elaborar um livro de texto para os seus alunos. Ao preparar o livro passou em revista as suas «fichas» sobre os elementos, deparando-se-lhe uma repetição periódica de propriedades, o que o motivou para a reflexão e pesquisa.

O seu livro «Princípios de Química» conheceu 8 edições em Russo e várias traduções em Inglês, Alemão e Francês.

Em Março de 1869 Mendeleev apresentou à Sociedade de Química Russa a comunicação «Acerca da Relação entre as propriedades dos Elementos e os seus Pesos Atómicos» [1] que apareceu transcrita em sinopse no Zeitschrift für Chemie, no Outono do mesmo ano.

Os elementos na Tabela de Mendeleev, Figura 1, estão organizados em linhas por ordem crescente do peso atómico. Interrompendo as linhas, ou PERÍODOS, no local apro-

\begin{tabular}{|c|c|c|c|c|c|c|c|}
\hline$\underset{n \in 0}{\text { Geuro I }}$ & $\begin{array}{c}\text { churo " } \\
- \\
\text { no }\end{array}$ & $\begin{array}{c}\text { GRUPO II } \\
- \\
\mathrm{n}, \mathrm{O}\end{array}$ & $\begin{array}{c}\text { GRUPO iv } \\
\text { RH, } \\
\text { RO, }\end{array}$ & $\begin{array}{c}\text { GAUPO V } \\
\mathrm{RH}_{0} \\
\mathrm{RaO}\end{array}$ & $\begin{array}{c}\text { GRUPO VI } \\
\text { NM, } \\
\text { NO, }\end{array}$ & $\begin{array}{c}\text { GRUPO Vu } \\
\mathrm{NH} \\
\mathrm{N}, \mathrm{O},\end{array}$ & $\begin{array}{c}\text { canupo vit } \\
\overline{\text { no, }}\end{array}$ \\
\hline \multicolumn{8}{|l|}{$\mathrm{H}=1$} \\
\hline$U=7$ & $\mathrm{Be}=9,4$ & $B=11$ & $C=12$ & $N=14$ & $0=16$ & $f=19$ & \\
\hline $\mathrm{Na}=23$ & $M g=24$ & $N=27.3$ & Si $=28$ & $P=r 1$ & $s=32$ & $a=35, s$ & \\
\hline$K=39$ & $C_{2}=40$ & $-=4$ & $n=48$ & $v=51$ & $\mathrm{Cr}=\mathrm{s2}$ & $M a=55$ & $\begin{array}{l}\mathrm{Fe}=56, \mathrm{Cl}_{0}=59 \\
\mathrm{Ni}=59, \mathrm{Cu}=63\end{array}$ \\
\hline$\left(C_{u}=6\right)$ & $Z n=65$ & --68 & $-=72$ & $A s=75$ & $\mathrm{Se}=78$ & $\mathrm{Br}=80$ & \\
\hline$R b=85$ & $5 r=87$ & I $Y_{\mathrm{r}}=8 \mathrm{~s}$ & $\mathrm{Zr}=90$ & $\mathrm{Nb}=94$ & $M_{0}=96$ & --100 & $\begin{array}{l}R u=106, R h=100 \\
P d=106, A_{B}=106\end{array}$ \\
\hline$\left(A_{E}=108\right)$ & $C d=112$ & $\ln =113$ & $S_{n}=118$ & $5 b=122$ & $\mathrm{Te}=12 \mathrm{~S}$ & $I=127$ & \\
\hline \multirow[t]{2}{*}{$C_{s}=133$} & $\mathrm{Ba}=137$ & $? D i=138$ & $P C e=140$ & & & & \\
\hline & & I $E r=178$ & $? \mathrm{~L}=180$ & $\mathrm{Ta}=182$ & $W=184$ & & $\begin{array}{l}s=195, \quad t r=19 \\
P t=198, \quad A u=19\end{array}$ \\
\hline \multirow[t]{2}{*}{$(A v=19)$} & $\mathrm{H}_{\mathrm{g}}=\mathbf{2 0 0}$ & $\mathrm{n}=206$ & $\mathrm{~Pb}=207$ & $B i=206$ & & & \\
\hline & & & $T h=213$ & & $\mathrm{U}=240$ & & \\
\hline
\end{tabular}

priado, a sua justaposição dá origem a colunas, ou GRUPOS, de forma que os elementos de cada grupo têm propriedades semelhantes.

A classificação de Mendeleev, tal como a de Newlands assentava na «Variação periódica das propriedades físicas e químicas dos elementos em função dos respectivos pesos atómicos",

Com este critério ambas apresentavam inversão de três elementos: Iodo-Telúrio; Árgon-Potássio e Cobalto-Níquel, distinguiam-se no entanto na medida em que a classificação de Mendeleev apresenatva espaços vazios que vieram a ser progressivamente ocupados por elementos até então desconhecidos e para os quais tinha previsto as características.

Percorrendo a sua Tabela iam-se encontrando periodicamente os elementos de uma mesma família, daí recebendo a designação de Tabela Periódica.

A Tabela publicada meses mais tarde por L. Meyer é praticamente idêntica [2].

Entretanto, descobertas sobre a existência de uma estrutura interna do átomo [3] forneceram as bases teóricas que justificariam a periodicidade dos elementos químicos como consequência dessa estrutura atómica.

Ao considerar unitária a carga do núcleo do átomo de hidrogénio, Moseley (1910) conclui que as cargas positivas dos núcleos de todos os outros átomos são múltiplos inteiros daquela, introduzindo assim o conceito de número atómico, correspondendo afinal ao número de ordem anteriormente atribuído por Mendeleev.

Também cerca de 1910, Sir E. Rutherford defendeu a pouca importância do peso atómico, para efeitos classificativos, pois isótopos, com peso atómico diferente, mas com o mesmo número atómico, têm essencialmente as mesmas propriedades.

O modelo de distribuição electrónica de Bohr [4] vem explicar a gradação das propriedades físicas e químicas das famílias de elementos, desde uns mais reactivos a outros mais inertes, tendo Bohr preferido uma organização em pirâmide, Figura 2.

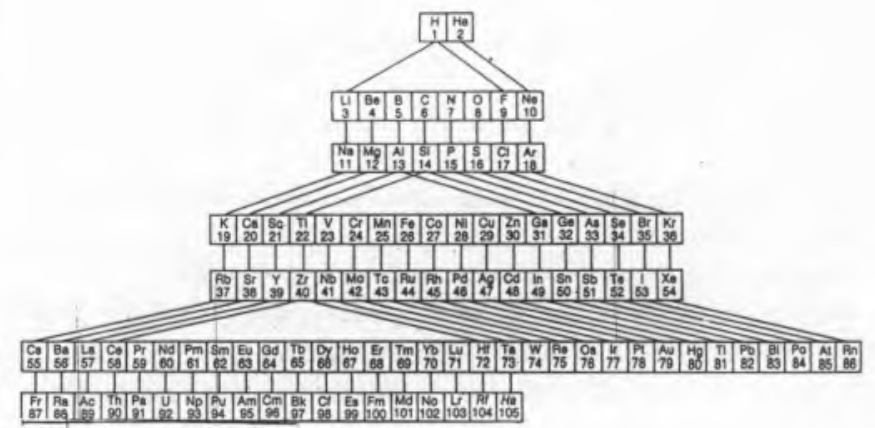

FIGURA 2

Tabela de Bohr em Pirâmide 
O desenvolvimento da teoria electrónica de valência e estrutura molecular com início no postulado da partilha de pares de electrões, por G.N. Lewis em 1916 também desempenhou parte importante na explicação da lei da periodicidade.

Outros avanços resultaram da formulação das regras gerais da Teoria Quântica de W. Wilson e A. Sommerfeld em 1916, a descoberta do princípio de exclusão de W. Pauli em 1925 , a descoberta do spin do electrão por G. Euhlenbeck e S. Goudsmit em 1925 e o desenvolvimento da Mecânica Quântica de W. Heisenberg e E. Schrödinger no mesmo ano.

Em 1922 verificaram-se algumas lacunas, restando por descobrir os elementos de número atómico 43, 61, 72, 75, 85, 87 e superior a 92 .

A partir da Tabela de Bohr, Deming [5] desenvolveu uma versão alargada da Tabela Periódica, Figura 3 com 18 grupos.

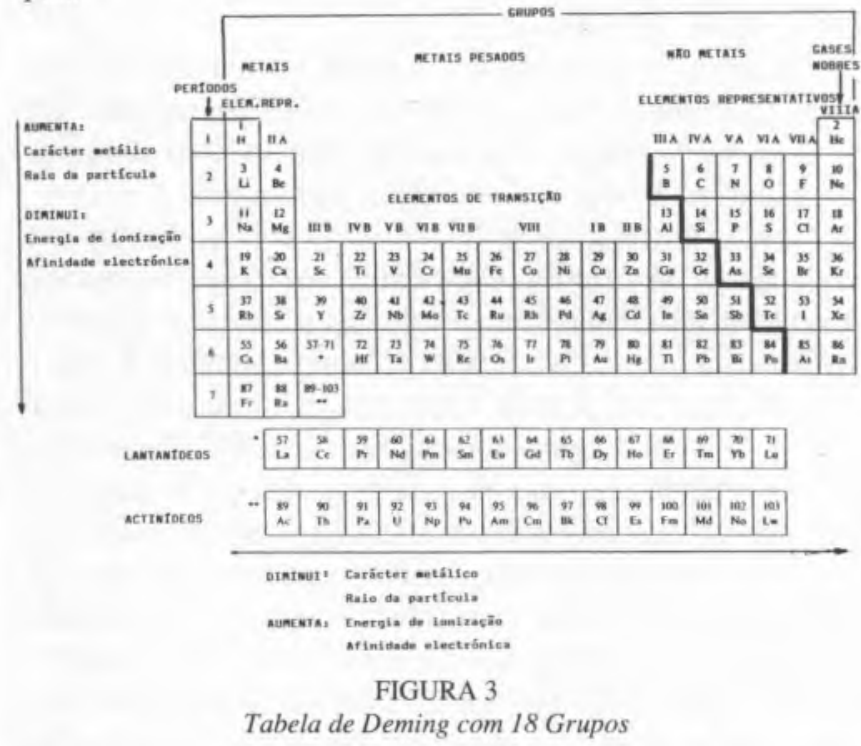

Este sistema periódico contém todos os elementos descobertos e os que vieram a ser progressivamente descobertos ou produzidos em reacções altamente energéticas por ordem dos números atómicos, organizada em sete peródos horizontais e dezoito grupos verticais. Os períodos têm extensões diferentes.

Há primeiro o período do hidrogénio com dois elementos, $\mathrm{H}$ 1 e He 2. Há depois dois períodos de oito elementos cada, o primeiro período curto de $\mathrm{Li} 3$ a Ne 10 e o segundo período curto de $\mathrm{Na} 11$ a $\mathrm{Ar} 18$.

Seguem-se dois períodos de 18 elementos cada, o primeiro período longo de K 19 a Kr 36 e o segundo longo de Rb 37 a Xe 54.

O período seguinte, longo de 32 elementos Cs 55 a Rn 86 é condensado em 18 colunas por omissão dos lantanídeos (ou lantanóides) desde Ce 58 a Lu 71 e dos actinídeos (ou actinóides) desde Th 90 a Lr 103, que são indicados separadamente na parte inferior, permitindo que os 18 resultantes, semelhantes aos correspondentes do $2 .^{\circ}$ e $3 .^{\circ}$ períodos longos, fiquem imediatamente abaixo. O último período está incompleto. As propriedades destes elementos indicam que será mais um segundo período longo de 32 elementos que ficaria completo no elemento 118 .

Convencionou-se designar os elementos a partir do elemento de número atómico 104 inclusive com base no nome latino que traduz o número de ordem,
Ex.: Unq 104 - U nil quadim

Unp 105 - U nil pentium

Unh 106 - U nil hexium

\section{Configuração}

electrónica;

Electrões de

valência

Período curto $\mathrm{H}, \mathrm{He}$ : 2 elementos $1 \mathrm{~s}$

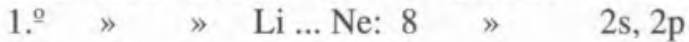

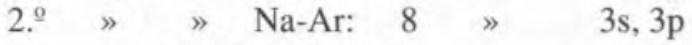

1..$^{\circ}$ longo K-Kr: 18 » $3 \mathrm{~d}, 4 \mathrm{~s}, 4 \mathrm{p}$

2. ${ }^{\circ} \quad$ Rb-Xe: 18 " $\quad 4 \mathrm{~d}, 5 \mathrm{~s}, 5 \mathrm{p}$

" " Cs-Rn: $32 \quad$ " $4 \mathrm{f}, 5 \mathrm{~d}, 6 \mathrm{~s}, 6 \mathrm{p}$

"incompleto Fr-118:32 » 5f, 6d, 7s, 7p

Os elementos dispostos na mesma coluna, ou grupo, apresentam configurações electrónicas semelhantes para os electrões periféricos, ou electrões de valência. Os dois primeiros grupos correspondem ao preenchimento de electrões de valência em orbitais s. Os dez grupos seguintes correspondem à ocupação progressiva de orbitais d. Os últimos 6 grupos correspondem à ocupação de orbitais p. Os elementos dos grupos s e p são conhecidos por elementos representativos e os elementos dos grupos d são usualmente designados por elementos de transição.

Para referenciar os grupos era usada a numeração romana: o número de um grupo indica o número de electrões de valência. Os dois primeiros grupos e os últimos seis recebiam adicionalmente a notação «A» ( o Grupo dos Gases Nobres, inicialmente localizado à esquerda da tabela por Deming com o número de ordem zero, foi posteriormente transferido para a direita recebendo a designação de Grupo VIII A).

Os grupos intermédios, Grupos de Transição, eram designados por «B» de tal forma que para um mesmo número houvesse correspondência entre óxidos do Grupo A e óxidos do Grupo B.

Os grupos encimados por Ferro, Cobalto e Níquel não receberam designação $\mathrm{A}$ ou $\mathrm{B}$.

Esta foi a versão divulgada ao longo de vários anos por Sargent-Welch Scientifica Company, Skikie, Illinois, USA e que cada membro da Sociedade Portuguesa de Química pode encontrar no verso do seu cartão de sócio!

Não pode no entanto dizer-se que a utilização de tal nomenclatura tenha sido generalizada pois verifica-se que particularmente as designações A e B foram largamente usadas e abusadas de forma arbitrária, quer em livro de texto, quer em artigos científicos. Um estudo sobre o assunto [6] revela que em 10\% dos artigos citados no Chemical Abstracts é inclusivamente quase impossível deduzir claramente sobre quais os elementos em causa.

De facto, por exemplo, em 1924, W.M. Welch Manufacturing Company publicou uma versão reduzida da Tabela Periódica, que se poderá atribuir a uma proposta de $\mathrm{H}$. Hubbard, ex-Secretário do National Bureau of Standards em que, nos grupos III a VII as designações A e B eram usadas exactamente de forma contrária à de Deming.

Também na Europa foi popular uma versão em que à numeração $\mathrm{I}_{\mathrm{A}}$ e $\mathrm{II}_{\mathrm{A}}$ dos metais alcalinos e alcalino terrosos se 
seguia $\mathrm{III}_{\mathrm{A}}, \mathrm{IV}_{\mathrm{A}}, \ldots, \mathrm{VIII}_{\mathrm{A}}$ desde $o$ Grupo do escândio aos do ferro, do cobalto e do níquel! Surgia em seguida $\mathrm{I}_{\mathrm{B}}$ e $\mathrm{II}_{\mathrm{B}}$ para cobre e zinco seguidos de $\mathrm{III}_{\mathrm{B}}, \mathrm{IV}_{\mathrm{B}}, \ldots, \mathrm{VII}_{\mathrm{B}}$ desde o boro, carbono, azoto, oxigénio, até aos halogéneos. Em 1970 a Comissão de Nomenclatura em Química Inorgânica da União Internacional de Química Pura e Aplicada (CNIC-IUPAC) [7] recomendava ainda uma sequência análoga mas utilizando algarismos árabes, omitidos no entanto os elementos dos três primeiros grupos.

A partir de 1980 a CNIC procurou resolver esta situação tendo chegado à recomendação publicada na edição de 1988 do «Nomenclature of Inorganic Chemistry (Part I)» (vulgo Livro Vermelho). Apesar de existirem publicados numerosos e diversificados sistemas de organização [8], a versão adoptada baseia-se numa proposta de A. Oländer de $1972 \mathrm{e}$ da qual ele tinha registado direitos de autor desde 1956, publicada a primeira vez em 1964. Nela, os grupos da Tabela Periódica começavam com o grupo dos Gases Nobres e recebiam a numeração seguida de 0 a 17 . A única alteração residiu em fazer desaparecer o grupo 0 passando os gases nobres para o fim com a numeração 18 , Figura 4 .

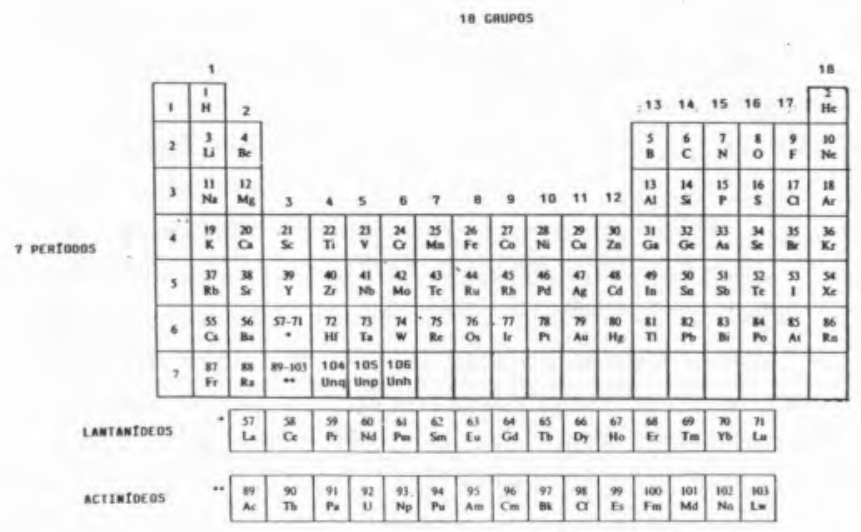

FIGURA 4

Tabela 1 a 18, actual recomendaçâo de CNOC-IUPAC

Em termos de números tudo se manteve, excepto para os elementos boro, carbono, azoto, oxigénio e flúor que eram os primeiros elementos dos Grupos $\mathrm{III}_{\mathrm{A}}$ a $\mathrm{VII}_{\mathrm{A}}\left(\right.$ ou $\mathrm{III}_{\mathrm{B}}$ a $\mathrm{VIII}_{\mathrm{B}}$ ?! ; ou $3_{\mathrm{B}}$ a $7_{\mathrm{B}}$ ?!) e passaram a pertencer aos grupos 13 a 17.

Esta proposta foi alvo de várias críticas, a maioria das quais se relacionava com a perda de correlação entre os números das colunas e os números de oxidação ou os números de electrões de valência.
Algumas objecções acompanhadas de propostas alternativas são tendentes a manter a distinção entre Grupos Principais e Grupos de Transição, por exemplo recorrendo às letras $\mathrm{M}$ (Main) e T (Transition) ou L (Left) e R (Right) que pecam pela falta de universalidade da língua inglesa).

Outra objecção parte dos químicos e físicos do estado sólido para quem a alusão aos semicondutores III/V se torna familiar. Não se vê qualquer conveniente em continuar a usar tal referência [9].

Foi no entanto eliminada a possibilidade de utilização arbitrária de A ou B para os grupos e a mesma correlação anterior pode ser facilmente encontrada à custa de uma pequena mnemónica, pois que um átomo quer de um grupo principal, quer de um grupo de transição tem um número de electrões de valência igual ao último dígito do número da coluna respectiva; (apenas os elementos do grupo 10 têm 10 electrões de valência).

Os electrões de um grupo representam a soma dos electrões $\mathrm{s}+\mathrm{p}+\mathrm{d}$ a seguir ao gás nobre anterior. A nova numeração tem ainda a vantagem de eliminar três grupos com a mesma designação VIII que passaram a ser os grupos 8,9 e 10 .

Os apoios à notação 1 a 18 foram no entanto largamente encorajadores e extensas listas de méritos foram salientados, acompanhados inclusivamente de sugestões de mnemónicas tendentes a facilitar a adopção do novo sistema [10,11], o que levou a CNIC a fazer a recomendação, sem no entanto deixar de incluir alguns comentários. A IUPC não faz leis, faz recomendações com base na experiência dos membros das suas comissões e apela à ampla discussão e comentário sobre este como sobre outros assuntos que são do interesse de todos os membros da comunidade dos químicos. A Comissão é de opinião que seja qual for o tempo necessário, recomendações sobre designações e formato da Tabela Periódica devem corresponder ao mais largo consenso possível, todos sendo convidados a apresentar as suas propostas à CNIC.

\section{Referências}

[1] D.I. Mendeleev, J. Russ. Chem. Ges., I, 60 (1869).

[2] L. Meyer, Liebigs Ann. Chem. Suppl., 7, 354 (1970).

[3] T. Bayley, J. Am. Chem. Soc., 20, 927, 935 (1898).

[4] N. Bohr, Z. Phys., 9, 1 (1922).

[5] H.G. Deming - General Chemistry, New York (1923).

[6] N.C. Fernelius e W.H. Powell, J. Chem. Educ., 59, 504 (1982).

[7] Nomenclature of Inorganic Chemistry, 2nd Edition, Butterworths, London (1970).

[8] The Periodic System of Chemical Elements, Ed. J.W. van Sprosen Elsevier, Amsterdam, 1969.

[9] D.H. Busch, Chemistry International, 9,49 (1987).

[10] N.E. Holden, Chem. Eng. News, 64, n. ${ }^{\circ} 115,3$ (1986). R.J. Tykodi, Chem. Eng. News, 64, n. $.15,3$ (1986).

[11] E. Fluck e K. Rumpf, Chemie in unserer Zeit, 20, 111 (1986). 
FEDERATION OF EUROPEAN

CHEMICAL SOCIETIES

Working Party in Chemical Education

\section{$1^{\text {st }}$ European Conference} on Research in Chemical

\section{Education}

\section{$1^{\text {st }}$ ECRIRE}

$1^{\text {ère }}$ Conférence Européenne sur la

Recherche en

Didactique de la Chimie

9 èmes JIREC

gèmes Journées de l'Innovation et

de la Recherche

dans l'Education en Chimie

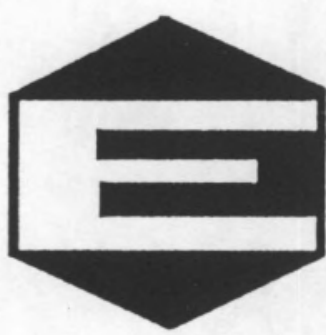

First Circular

Première Circulaire

25 - 28 / 08 / 1992

Montpellier

France

Organisées par / Organized by Société Française de Chimie

\section{RESEARCH IN CHEMICAL EDUCATION}

Conference Aims

Representations - Conceptions

Evaluation

Trends in didactics

Problem solving

Experimental teaching

Computer-assisted teaching

New technologies

\section{Objectives}

To describe the state of chemical education research and to help chemists develop this research

To develop a european cooperation for the defense and survival of didactics

To demonstrate interest of chemical education research

To involve more and more chemists in this research

To improve chemistry teaching

To single out priorities

\section{Language \\ English - French}

Registration fees: 1500 FF

Members FEC: 1000 FF

Secondary school teachers: $400 \mathrm{FF}$

Preregistration

01/15/92

Registration

$04 / 01 / 92$

Submission of abstracts $\quad 04 / 01 / 02$

Full papers 


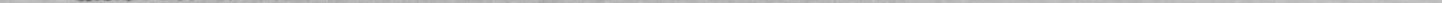

